\title{
Inhibition of formyl peptide receptor in high-grade astrocytoma by CHemotaxis Inhibitory Protein of S. aureus
}

\author{
J C Boer ${ }^{1}$, U M Domanska ${ }^{1}$, H Timmer-Bosscha ${ }^{1}$, I G J Boer ${ }^{2}$, C J C de Haas ${ }^{2}$, J V Joseph ${ }^{1}$, F A E Kruyt ${ }^{1}$, \\ E G E de Vries ${ }^{1}$, W F A den Dunnen ${ }^{3}$, J A G van Strijp ${ }^{2}$ and A M E Walenkamp*,1
}

${ }^{1}$ Department of Medical Oncology, University Medical Center Groningen, University of Groningen, Hanzeplein 1, 9700 RB Groningen, The Netherlands; ${ }^{2}$ Department of Medical Microbiology, University Medical Center Utrecht, University of Utrecht, Heidelberglaan 100, 3584 CX Utrecht, The Netherlands and ${ }^{3}$ Department of Pathology and Medical Biology, University Medical Center Groningen, University of Groningen, Hanzeplein 1, 9700 RB Groningen, The Netherlands

Background: High-grade astrocytomas are malignant brain tumours that infiltrate the surrounding brain tissue and have a poor prognosis. Activation of formyl peptide receptor (FPR1) on the human astrocytoma cell line U87 promotes cell motility, growth and angiogenesis. We therefore investigated the FPR1 inhibitor, Chemotaxis Inhibitory Protein of S. aureus (CHIPS), as a potential antiastrocytoma drug.

Methods and results: FPR1 expression was studied immunohistochemically in astrocytomas WHO grades I-IV. With intracellular calcium mobilisation and migration assays, human ligands were tested for their ability to activate FPR1 on U87 cells and on a cell line derived from primary astrocytoma grade IV patient material. Thereafter, we selectively inhibited these ligand-induced responses of FPR1 with an anti-inflammatory compound called Chemotaxis Inhibitory Protein of S. aureus (CHIPS). U87 xenografts in NOD-SCID mice served to investigate the effects of CHIPS in vivo. FPR1 was expressed in 29 out of 32 (90\%) of all grades of astrocytomas. Two human mitochondrial-derived formylated peptides, formyl-methionil-leucine-lysine-isoleucine-valine (fMLKLIV) and formyl-methionil-methionil-tyrosine-alanine-leucine-phenylalanine (fMMYALF), were potent activators of FPR1 on tumour cells. Ligand-induced responses of FPR1-expressing tumour cells could be inhibited with FPR1 inhibitor CHIPS. Treatment of tumour-bearing mice with CHIPS slightly reduced tumour growth and improved survival as compared to non-treated animals $(P=0.0019)$.

Conclusion: Targeting FPR1 with CHIPS reduces cell motility and tumour cell activation, and prolongs the survival of tumourbearing mice. This strategy could be explored in future research to improve treatment results for astrocytoma patients.

Astrocytoma is a malignant neoplasm arising from astrocytes within the central nervous system. The tumour aggressiveness ranges from slowly growing low-grade tumours to rapidly growing high-grade tumours. World Health Organisation (WHO) grade IV astrocytoma, generally referred to as glioblastoma (GBM), is the most frequently occurring primary malignant brain tumour in adults. Median survival after diagnosis is $\sim 7$ years for patients with grade II astrocytoma (Louis et al, 2007), whereas the majority of grade IV astrocytoma patients have a median survival of only 12-15 months (Wen and Kesari, 2008). The current treatment of astrocytoma consists of surgery followed by radiotherapy and chemotherapy. However, this strategy does not target tumour cell migration into surrounding brain tissue, which is a fundamental feature of astrocytoma and is the major reason for treatment failure. Therefore, novel drugs that would target tumour spread are urgently needed. 
Tumour cell migration and leucocyte trafficking have many similarities (Zlotnik, 2006). Mechanisms involved in leucocyte migration, such as the expression of $G$ protein-coupled receptors (GPCRs), could offer important clues for drug development. One of such GPCR, formyl peptide receptor (FPR1), was initially identified to mediate leucocyte migration and was characterised by its ability to bind bacterial-derived chemotactic $N$-formyl peptides, such as $N$-formyl-methionyl-leucyl-phenylalanine (fMLF) (Schiffmann et al, 1975; Durstin et al, 1994).

Binding of the bacterial peptide fMLF to its receptor FPR1 causes the activation of phagocytic leucocytes, essential for the innate host defence against microbial infection (Schiffmann et al, 1975). More recently, FPR1 was found to be present on tumour cells and has been identified as a factor contributing to their malignant behaviour (Zhou et al, 2005; Huang et al, 2010).

Agonist binding to FPR1 in high-grade astrocytoma cells elicits a cascade of signal transduction pathways that involve protein kinase B (Akt), phosphatidylinositol 3-kinase (PI3K), mitogenactivated protein kinases (MAPK) and the transcription factor nuclear factor $-\kappa \mathrm{B}$, which are linked with tumour-promoting cellular processes such as proliferation, migration and angiogenesis (Vivanco and Sawyers, 2002; Zhou et al, 2005; Yao et al, 2008a).

Mitochondria contain formylated peptides that, analogously to fMLF, have affinity for FPR1, as shown in promyelocytic HL-60 cells (Rabiet et al, 2005). Moreover, the supernatant of necrotic astrocytoma cells contains agonists capable of inducing FPR1 activation, followed by a robust intracellular calcium mobilisation in U87 astrocytoma cells (Zhou et al, 2005). Therefore, we hypothesised that FPR1 activation in astrocytoma cells can be induced by mitochondrial peptides present in the necrotic cell debris, which serve as natural ligands for FPR1.

The FPR1 inhibitor Chemotaxis Inhibitory Protein of S. aureus (CHIPS) is an anti-inflammatory compound secreted by $S$. aureus (de Haas et al, 2004). This virulence factor directly binds to FPR1 and $\mathrm{C} 5 \mathrm{a}$ receptor $(\mathrm{C} 5 \mathrm{aR})$, inhibiting neutrophil responses to bacterial infection (Postma et al, 2004). The potency of CHIPS to inhibit FPR1, together with the contribution of FPR1 function to malignant tumour behaviour, prompted us to test CHIPS as a novel inhibitor of tumour cell activation and migration.

In this study, we investigated FPR1 expression in astrocytomas WHO grade I-IV. Furthermore, we tested relevant human mitochondrial ligands for their capacity to activate FPR1 on U87 astrocytoma cells and then studied the possibilities of selectively inhibiting these effects with CHIPS. Finally, we investigated the effect of CHIPS treatment in a human tumour-bearing mouse model.

\section{MATERIALS AND METHODS}

Reagents. L-glutamine and gentamicin were obtained from Invitrogen Life Technologies (Bleiswijk, The Netherlands). fMLF, ionomycin calcium salt, porcine skin gelatin and Corning Transwell polycarbonate membrane inserts $(8.0 \mu \mathrm{m}$ pore size $)$ and 3-(4,5-dimethylthiazol-2-yl)-2,5-diphenyltetrazolium bromide (MTT) were purchased from from Sigma-Aldrich (Zwijndrecht, the Netherlands). Fluorescein isothiocyanate (FITC)-labelled fMLF and fluo-3 acetoxymethyl ester (Fluo-3-AM) were purchased from Molecular Probes (Leiden, The Netherlands) and mitochondrial peptides fMLKLIV and AMMYALF from Isogen Life Science (de Meern, The Netherlands). Coomassie brilliant blue G-250 was obtained from Bio-Rad Laboratories (Veenendaal, The Netherlands), Immobilon-P membranes from Millipore Corporation (Amsterdam, The Netherlands), anti phospho-Akt (Thr308) (C31E5E) rabbit monoclonal antibody (mAb, no. 2965), anti phospho-p44/42 MAPK (Erk1/2) (Thr202/Tyr204) (D13.14.4E)
$\mathrm{XP}$ rabbit (mAb, \#4370) and rabbit polyclonal antibody against cleaved caspase-3 (\#9661S) were purchased from Cell Signalling Technology (Leiden, The Netherlands). Polyclonal rabbit antibody against FPR1 (\# ab12990) was purchased from Abcam (Cambridge, UK), Lumi-light ${ }^{\text {plus }}$ Western blotting substrate and Pwo DNA polymerase from Roche Diagnostics GmBH (Mannheim, Germany). Rabbit polyclonal antibody against glucose transporter 1 (GLUT-1), horse radish peroxidase (HRP)-labelled goat anti rabbit and goat anti mouse antibodies were obtained from Dako (Glostrup, Denmark). Protease inhibitor cocktail EDTA-free $100 \times$ and Halt phosphatase from Thermo Scientific (Breda, The Netherlands). Mammalian Protein Extraction Reagent (MPER) was obtained from Thermo Scientific (Etten-Leur, The Netherlands). Quantikine human VEGF ELISA kit was purchased from R\&D systems (Abingdon, UK). The $70 \mu \mathrm{m}$ pore size nylon cell strainer was purchased from Beckton Dickinson (Breda, The Netherlands).

Cells. The human astrocytoma cell line U87 was purchased from the ATCC (HTB-14). A newly generated cell line was derived from a 44-year old female patient diagnosed with astrocytoma grade IV. Primary material was obtained after approval and following the ethical guidelines of the Institutional Review Board of the UMCG. Resected tumour tissue was mechanically dissociated, followed by incubation with trypsin at $37^{\circ} \mathrm{C}$ for $15 \mathrm{~min}$, repeated pipetting and filtration through a $70 \mu \mathrm{M}$ cell strainer to obtain single cells. Cells were centrifuged, washed with $\mathrm{PBS}$ (phosphate buffered saline, $2.7 \mathrm{~mm} \mathrm{KCl}, 1.8 \mathrm{~mm} \mathrm{KH}_{2} \mathrm{PO}_{4}, 137 \mathrm{~mm} \mathrm{NaCl}, 10.1 \mathrm{~mm} \mathrm{Na}_{2} \mathrm{HPO}_{4}$, $\mathrm{pH}=7.4$ ) and resuspended in culture medium for propagation. The expression of neuronal and glial markers was confirmed by immunofluorescence microscopy and Western blotting. (Supplementary Figure S1A and S1B). Both U87 and the patient derived cell lines were grown in cell culture flasks pre-coated with $1 \%$ gelatin from porcine skin and maintained in Dulbecco's modified Eagle Medium high glucose (DMEM-H) supplemented with $10 \%$ foetal calf serum (FCS). The human promonocytic cell line U937 and its transfected subline U937-FPR were kindly provided by Dr Eric R Prossnitz (University of New Mexico, Albuquerque, NM, USA). Cells were maintained in Roswell Park Memorial Institute medium 1640 (RPMI 1640) containing 10\% FCS, $1 \%$ L-glutamine and $10 \mu \mathrm{g} \mathrm{ml}^{-1}$ gentamicin. All cell lines were cultured at $37^{\circ} \mathrm{C}$ in a humidified atmosphere with $5 \% \mathrm{CO}_{2}$.

Expression of Chemotaxis Inhibitory Protein of $S$. aureus (CHIPS) in E. coli. CHIPS was expressed in E. coli, as described by (de Haas et al, 2004). Briefly, the chp gene was amplified by PCR on chromosomal DNA of $S$. aureus (strain Newman) using Pwo DNA polymerase. The PCR product was cloned into the pTrcHISB vector (Invitrogen, Bleiswijk, The Netherlands) directly downstream of the enterokinase cleavage site. The vector was transformed into TOP10 E. coli, and recombinant CHIPS was expressed and purified according to the manufacturer's instructions (Invitrogen, Bleiswijk, The Netherlands).

Ligand binding assay. $5 \times 10^{6}$ U87 cells expressing FPR1 were incubated for $45 \mathrm{~min}$ on ice in RPMI 1640 with $1 \times 10^{-5} \mathrm{M}$ fluorescent formylated peptide ligand (fMLF-FITC). After incubation, the cells were washed twice, and the fluorescence of the cells was measured by flow cytometry (FACSCalibur; Becton Dickinson, Franklin Lanes, NJ, USA). Data were analysed with WinList software (Verity Software House, Topsham, ME, USA).

CHIPS-FITC binding. CHIPS was labelled with FITC as previously described (Postma et al, 2004). FPR1-expressing U87 cells were incubated with increasing concentrations of CHIPSFITC $\left(3,10\right.$ and $\left.20 \mu \mathrm{g} \mu \mathrm{l}^{-1}\right)$ for $30 \mathrm{~min}$ at $37^{\circ} \mathrm{C}$ in RPMI 1640 containing $0.05 \%$ human serum albumin (RPMI/HSA). Subsequently, fluorescence of the cells was measured by flow cytometry and analysed as described above. 
Blocking cellular fMLF-FITC binding with CHIPS. $5 \times 10^{5}$ U87 or U937-FPR cells were preincubated with RPMI 1640 medium only or with different CHIPS concentrations ( 1 and $100 \mu \mathrm{g} \mathrm{ml}^{-1}$ ) for $15 \mathrm{~min}$ at $4{ }^{\circ} \mathrm{C}$. Subsequently cells were incubated for $45 \mathrm{~min}$ at $4{ }^{\circ} \mathrm{C}$ in RPMI 1640 medium with $5 \times 10^{-6} \mathrm{M}$ (U87) or $10^{-7} \mathrm{M}$ (U937-FPR) fluorescent formylated peptide ligand (fMLF-FITC). After incubation, the cells were washed twice and fluorescence was measured by flow cytometry as described above.

$\mathrm{Ca}^{2+}$ mobilisation assay. $5 \times 10^{6}$ U87, U937-FPR and the patient-derived cells were centrifuged twice $(10 \mathrm{~min}, 250 \times \mathrm{g}$, at $4{ }^{\circ} \mathrm{C}$ ) in $\mathrm{RPMI} / \mathrm{BSA}$ and resuspended in $1 \mathrm{ml}$ of RPMI/BSA. Intracellular calcium was labelled by adding $2 \mu \mathrm{l}$ Fluo-3-AM to the cell suspension. Cells were incubated for $20 \mathrm{~min}$ at room temperature on a shaking table, centrifuged twice and suspended in RPMI/BSA. Thereafter, patient-derived cells were incubated with $1 \mu \mathrm{g} \mathrm{ml}^{-1}$ CHIPS and U87 and U937-FPR1 cells were incubated with $0,0.01,0.1$ or $1 \mu \mathrm{g} \mathrm{ml}^{-1}$ CHIPS for $15 \mathrm{~min}$ at room temperature. Samples were analysed by flow cytometry as described above. First, the background fluorescence of cells was measured. Subsequently, cells were stimulated with fMMYALF, fMLKLIV or fMLF peptides in concentrations ranging from $10^{-8}$ $10^{-5} \mathrm{M}$ for U87, U937-FPR and patient-derived cells, or with ionomycin as a positive control. Data were calculated as the percentage of maximal calcium mobilisation using the following formula: $\left(\left(\mathrm{MF}_{\text {sample }}-\mathrm{bgF}_{\text {sample }}\right) /\left(\mathrm{MF}_{\max }-\mathrm{bgF}_{\max }\right)\right) \times 100 \%$, in which $\mathrm{MF}=$ mean fluorescence with stimulation and $\mathrm{bgF}=$ background fluorescence without stimulation. For the maximal MF $\left(\mathrm{MF}_{\max }\right)$, the $\mathrm{MF}$ value of cells stimulated with the highest concentration of stimulus was used.

Chemotaxis assay (Transwell). Transwell inserts with $8 \mu \mathrm{m}$ pores were coated with $50 \mu \mathrm{g} \mathrm{ml}^{-1}$ collagen type I and incubated for $1 \mathrm{~h}$ at $37^{\circ} \mathrm{C}$. Transwell inserts and lower wells were blocked with $1 \%$ BSA/PBS overnight at $4{ }^{\circ} \mathrm{C}$; thereafter, blocking medium was removed and lower wells were loaded with $300 \mu \mathrm{l}$ of fMLF, fMMYALF or PMLKLIV in DMEM $+0.5 \%$ BSA in concentrations ranging from $10^{-7} \mathrm{M}$ to $10^{-10} \mathrm{M}$.

U87 or U937-FPR1 cells were serum starved for 2 days. All steps were performed respectively in serum-free DMEM-H and RPMI 1640 medium. After this, $6 \times 10^{5}$ cells per $\mathrm{ml}$ were harvested and incubated for $15 \mathrm{~min}$ with control medium or $10 \mu \mathrm{g} \mathrm{ml}^{-1}$ of CHIPS medium. Subsequently, $1 \times 10^{5}$ cells per well in $150 \mu \mathrm{l}$ serum-free DMEM-H or RPMI 1640 medium were loaded at the top of Transwell inserts.

Cells were allowed to migrate towards concentrations of fMLF or serum-free DMEM-H (U87) or fMLF, fMMYALF, fMLKLIV or serum-free RPMI 1640 medium (U937-FPR1) for $4.5-5.5 \mathrm{~h}$ at $37^{\circ} \mathrm{C}$. After migration, the top surface of the Transwell inserts was cleaned with a cotton swap wetted in PBS. The cells on the bottom surface of the Transwell insert were fixed with $75 \%$ methanol/25\% acidic acid for $20 \mathrm{~min}$ and stained with $0.25 \%$ Coomassie blue in $45 \%$ methanol $/ 10 \%$ acetic acid for $20 \mathrm{~min}$. Excessive staining of Coomassie blue was removed by washing the Transwell with demi water. Migrated cells were identified under the microscope at $40 \times$ magnification by counting 10 fields of view in the middle of the filter from left to right.

Immunoblotting. Western blot analysis of Akt, phosphorylated Akt (phospho-Akt) and phosphorylated extracellular signalregulated kinases 1 and 2 (phospho-ERK1/2) was performed. U87 cells $\left(1 \times 10^{6}\right)$ were seeded and starved for $24 \mathrm{~h}$, subsequently cells were preincubated with culture medium or with $1 \mu \mathrm{g} \mathrm{ml} l^{-1}$ CHIPS for $20 \mathrm{~min}$ at room temperature. Thereafter, cells were treated with buffer or different concentrations of fMLF $\left(10^{-5} \mathrm{M}\right.$, $10^{-7} \mathrm{M}$ and $10^{-8} \mathrm{M}$ ) for $30 \mathrm{~min}$. Cells were then lysed in MPER including 1:100 protease and 1:100 phosphatase inhibitors, sonicated for $3 \mathrm{~s}$ and boiled for $5 \mathrm{~min}$. Immunoblot analysis of phosphorylated ERK1/2 and Akt and total Akt was performed. Cell lysates were prepared with sample buffer $(25 \mathrm{~mm}$ Tris- $\mathrm{HCl}, 5 \%$ glycerol, $1 \%$ SDS, and $0.05 \%$ bromophenol blue, $\mathrm{pH}=6.8$ ). Proteins were separated on $10 \%$ polyacrylamide gel (SDS-PAGE) and transferred onto Immobilon-P membranes. The membranes were blocked by incubation in tris-buffered saline (TBS) with $0.01 \%$ Tween-20 (TBST) and 5\% BSA for $3 \mathrm{~h}$ at room temperature and then incubated overnight at $4{ }^{\circ} \mathrm{C}$ with primary antibodies against Akt, phospho-Akt and phospho-ERK1/2 in TBST. After incubation with a horseradish peroxidase-conjugated secondary antibody, the protein bands were detected. Enhanced chemiluminescence with Lumi-light ${ }^{\text {plus }}$ was used for final signal detection.

MTT assay. U87 cells (4000 cells per well) were plated in 96-well plates in $200 \mu \mathrm{l}$ of the culture medium. After $1 \mathrm{~h}$, fMLF, fMLKLIV or fMLALF $\left(0,10^{-5} \mathrm{M}, 10^{-6} \mathrm{M}, 10^{-7} \mathrm{M}, 10^{-8} \mathrm{M}\right.$ or $\left.10^{-9} \mathrm{M}\right)$ was added to each well. After 1, 2, 3, 4, 5 or 6 days of incubation with or without fMLF, cell proliferation was assessed by MTT assay as described previously (de Vries et al, 1989).

Enzyme linked immunosorbent assay (ELISA). U87 cells $\left(0.4 \times 10^{6}\right.$ per well $)$ were seeded in a 6 -well plate and preincubated with culture medium or with $10 \mu \mathrm{g} \mathrm{ml}^{-1}$ CHIPS for $15 \mathrm{~min}$. Cells were treated with fMLF $\left(10^{-7} \mathrm{M}\right)$ for $24 \mathrm{~h}$, supernatant was collected, frozen for VEGF-A analyses and cell numbers were counted. Quantification of VEGF-A protein by ELISA was performed by using the Quantikine Human VEGF ELISA kit according to the manufacturer's instructions. Results were calculated as pg of VEGF-A per $10^{6}$ cells and displayed as normalised values against control (100\%) with standard deviations.

Animal model. Male, nonobese diabetic (NOD)/severe combined immune deficient (SCID) mice (6-8 weeks old) were purchased from Harlan Laboratories (Horst, The Netherlands). Fourteen mice were preirradiated with $2 \mathrm{~Gy}$ and subcutaneously implanted in the dorsal flank with $5 \times 10^{6}$ U87 cells in $100 \mu$ l PBS. Animals were randomly divided into a treatment group and a control group. Starting from day one after tumour cell injection, the animals designated to the treatment group received daily intraperitoneal injections with $1.2 \mathrm{mg} \mathrm{kg}^{-1}$ CHIPS (in a total volume of $100 \mu \mathrm{l}$ PBS) and animals assigned to the control group received PBS intraperitoneally in an equal volume. Tumour size was calculated with the formula: $L w^{2} / 2$, where $L$ represents the length of the tumours in millimetres and $w$ the width in millimetres. The animals were killed after they reached the humane endpoint (tumour size $>2 \mathrm{~cm}^{3}$ ), and the tumours were excised, dissected into two pieces, snap frozen or formalin-fixed and paraffinembedded.

$5 \mu \mathrm{m}$ thick sections of paraffin-embedded tumours were subjected to haematoxylin and eosin (H\&E) staining and immunohistochemistry was performed to visualise cleaved caspase-3 as an apoptotic marker. All experimental procedures were approved by the Institutional Animal Care and Use Committee (IACUC) and conducted in accordance with institutional ethical regulations for experimental animal care.

Human tissue collection. $4 \mu \mathrm{m}$ cryosections of brain tumour specimens of 32 patients diagnosed between 2007 and 2010 with grade I-IV astrocytoma were retrieved from the tissue bank at the Department of Pathology of the University Medical Center Groningen. Tumours were histologically classified with $\mathrm{H} \& \mathrm{E}$ staining according to the criteria of the WHO (Louis et al, 2007). Tissue samples with representative histological characteristics and a sufficient amount of tissue for analyses were included. Tumour tissues were numerically tagged, based on a national coding system. According to Dutch law, no further Institutional Review and Board approval was required (www.federa.org). Eight pilocytic astrocytomas WHO grade I, 8 diffuse astrocytomas WHO 
grade II, 8 anaplastic astrocytomas WHO grade III and 8 astrocytoma WHO grade IV were stained for FPR1 expression.

Immunohistochemistry and staining evaluation. Both paraffinembedded xenografts from the animal study and frozen human tissues were stained immunohistochemically according to standard protocols. The paraffin-embedded tissue was first deparaffinised with xylol, followed by ethanol and transferred to citrate buffer $\mathrm{pH}=6.0$, heated for $15 \mathrm{~min}$ at $95^{\circ} \mathrm{C}$ and cooled to RT. Following steps were the same for frozen and paraffin-embedded specimens. Slides were washed with PBS, blocked with $0.33 \% \mathrm{H}_{2} \mathrm{O}_{2} / \mathrm{PBS}$ buffer for $30 \mathrm{~min}$ at room temperature and washed again with PBS. Subsequently, the sections were incubated for $1 \mathrm{~h}$ at room temperature with polyclonal primary antibodies against cleaved caspase-3, GLUT-1 or FPR1 diluted in 1\% BSA/PBS. Tumour sections were then washed with PBS and incubated with peroxidase-coupled goat antirabbit secondary antibody in $1 \%$ $\mathrm{BSA} / \mathrm{PBS}+1 \% \mathrm{AB}$ serum for $30 \mathrm{~min}$. After washing with PBS, the slides were incubated with peroxidase-coupled antigoat tertiary antibody in $1 \% \mathrm{BSA} / \mathrm{PBS}+1 \%$ human $\mathrm{AB}$ serum for $30 \mathrm{~min}$ followed by $3,3^{\prime}$-diaminobenzidine (DAB) treatment for $10 \mathrm{~min}$ and rinsed with tap water. Counter staining was performed with heamatoxylin for $2 \mathrm{~min}$ and washed with tap water. Paraffinembedded U937-FPR cells served as positive control for FPR1 staining. For all sections, omission of the primary antibody served as a negative control.

Staining evaluation was performed by double-blind scoring using Olympus WH10 $\times-\mathrm{H} / 22$ grid by scoring on high power field (ocular $10 \times$ and objective $40 \times$ ). Quantitative analysis was performed by calculating the percentage of positive cells on three high magnification fields per tumour slide. Subsequently, the antigenic load was calculated as described previously (Kuijlen et al, 2006), by multiplying the percentage of positive cells by their semiquantitative score. Semiquantitative score ranged in scale from 0 to 4 , defined as 0 when no staining, 1 as low intensity staining, 2 as moderate staining, 3 as high intensity staining and 4 as exceptionally intense staining.

For the evaluation of cleaved caspase-3 levels in U87 xenografts, a distinction was made between hypoxic and normoxic areas. As hypoxic areas strongly correlate with the expression of hypoxia marker GLUT-1 (Macheda et al, 2005; Liao et al, 2007), the regions located adjacently to the necrotic areas were defined as hypoxic zones and positive for GLUT-1. The normoxic areas were defined as the regions surrounding the hypoxic areas containing viable cells and no GLUT-1 expression (data not shown). Both normoxic and hypoxic areas were analysed (Sie et al, 2009).

Statistical analyses. All statistical analysis were performed with GraphPad Prism 5 (GraphPad Prism Software Inc., San Diego, CA, USA). Statistical significance was set at $P<0.05$. Kruskal-Wallis one-way analysis of variance was used (followed by Dunn's multiple comparison post-test) to assess differences in medians of antigenic load, percentage of positive cells between tumour grades. Data are expressed as median with confidence interval (CI) of $95 \%$. A non-parametric Student's $t$-test was performed to asses differences in VEGF-A expression. Data analyses on animal survival was performed using a log-rank (Mantel-Cox) test.

\section{RESULTS}

FPR1 is expressed in all grades of astrocytoma specimens. FPR1 expression was observed in all astrocytomas except for one pilocytic astrocytoma (WHO grade I), one diffuse astrocytoma (WHO grade II) and one glioblastoma (WHO grade IV). Low grade diffuse astrocytomas are often characterised by fibrillary astrocytes with irregular and elongated nuclei embedded in a fibrillary matrix (Miller and Perry, 2007). FPR1 expression in grade
I astrocytomas was less pronounced on the cell membrane, whereas it was highly expressed in the fibrillary structures (Figure 1A). In contrast, on grade-II astrocytoma specimens, mainly the membrane FPR1 expression was observed, mostly with intermediate staining intensity (Figure 1B). In higher grade astrocytomas with bulky cytoplasm, a weak cytoplasmic and strong perinuclear staining was observed (Figure $1 \mathrm{C}$ and $\mathrm{D}$ ). In grade I and II astrocytomas, $>70 \%$ of tumour cells were FPR1-positive with a homogeneous cytoplasmic staining pattern with moderate to high staining intensity (Figure $1 \mathrm{~A}$ and $\mathrm{B}$ ). In grade III astrocytomas (Figure 1C), 70\% of tumour cells were FPR1-positive, but the expression pattern was less homogeneous, although with high intensity. In grade IV astrocytomas, $40 \%$ of tumour cells were FPR1-positive. One grade-IV astrocytoma showed exceptionally high staining intensity, whereas the other seven showed strong positivity. The distribution of the staining throughout the tissue was heterogeneous (Figure 1D). The percentage of positive cells in grade IV $(33 \%)$ astrocytomas was lower $(P<0.05)$ than grade I $(59 \%)$ and grade III (63\%) astrocytomas (Figure 1E). The antigenic load of grade IV astrocytomas was also lower compared with other grades. However, only the difference in antigenic load between grade III and grade IV astrocytomas was significant $(P<0.05)$.

fMLF and CHIPS bind to FPR1-expressing U87. With the FPR1 ligand binding assay, we showed that fMLF-FITC binds to the FPR1 expressed on U87 (Figure 2A). We subsequently performed the CHIPS-FITC binding assay and found a dose-dependent binding of CHIPS-FITC to U87-FPR1-expressing cells (Figure 2B).

CHIPS blocks fMLF-FITC binding to U87 and U937-FPR1 cells. We investigated the ability of CHIPS to block fMLF-FITC binding to FPR1 on U87 and U937-FPR cells. Preincubation with 1 and $100 \mu \mathrm{g} \mathrm{ml}^{-1}$ CHIPS resulted in dose-dependent inhibition of fMLF-FITC binding on U937-FPR cells (Figure 2C). In U87 cells, $100 \mu \mathrm{g} \mathrm{ml}^{-1}$ of CHIPS resulted in $81 \pm 16 \%$ inhibition of fMLFFITC binding, whereas this effect was not observed after preincubation with $1 \mu \mathrm{g} \mathrm{ml}^{-1}$ CHIPS (Figure 2D).

CHIPS inhibits formylated peptide-induced calcium mobilisation. The hallmark of chemokine receptor activation is a rapid and transient increase in the free intracellular calcium level upon ligand binding. This signalling pathway was used to examine whether CHIPS-FITC not only binds FPR1, as demonstrated with the CHIPS-FITC binding assay, but also inhibits the activation by its natural ligand PMLF and mitochondrial peptides PMMYALF and fMLKLIV. U87 cells responded to increasing amounts of fMLF in a dose-dependent manner with intracellular calcium mobilisation (Figure 3A, control line). We could decrease calcium mobilisation up to $60 \%$ with $1 \mu \mathrm{g} \mathrm{ml}^{-1}$ CHIPS in U87 cells compared with control cells (Figure 3A). Similarly, U937-FPR cells showed intracellular calcium release following stimulation with fMLF (Figure $3 \mathrm{~B}$ ) and with both mitochondrial peptides fMLKLIV (Figure 3C) and fMMYALF (Figure 3D). Treatment with increasing CHIPS concentrations caused a dose-dependent inhibition of calcium mobilisation (Figure 3B-D). Finally, we used patient-derived cells that responded in a dose-dependent manner with intracellular calcium release. This could be decreased up to $70 \%$ with $1 \mu \mathrm{g} \mathrm{ml}^{-1}$ CHIPS (Supplementary Figure 1).

Overall, with the intracellular calcium mobilisation assay, we showed that not only fMLF, but also PMLKLIV and PMMYALF are agonists for FPR1 as demonstrated in both U937-FPR1 and U87 cells. Moreover, CHIPS can potently inhibit FPR1-mediated calcium mobilisation in U937-FPR, U87 and patient-derived cell lines.

CHIPS inhibits formylated peptides-induced migration of U87 and U937-FPR1 cells but not proliferation of U87 cells. We investigated the ability of CHIPS to inhibit the AMLF, fMMYALF and fMLKLIV-induced migration and proliferation of U87 and 

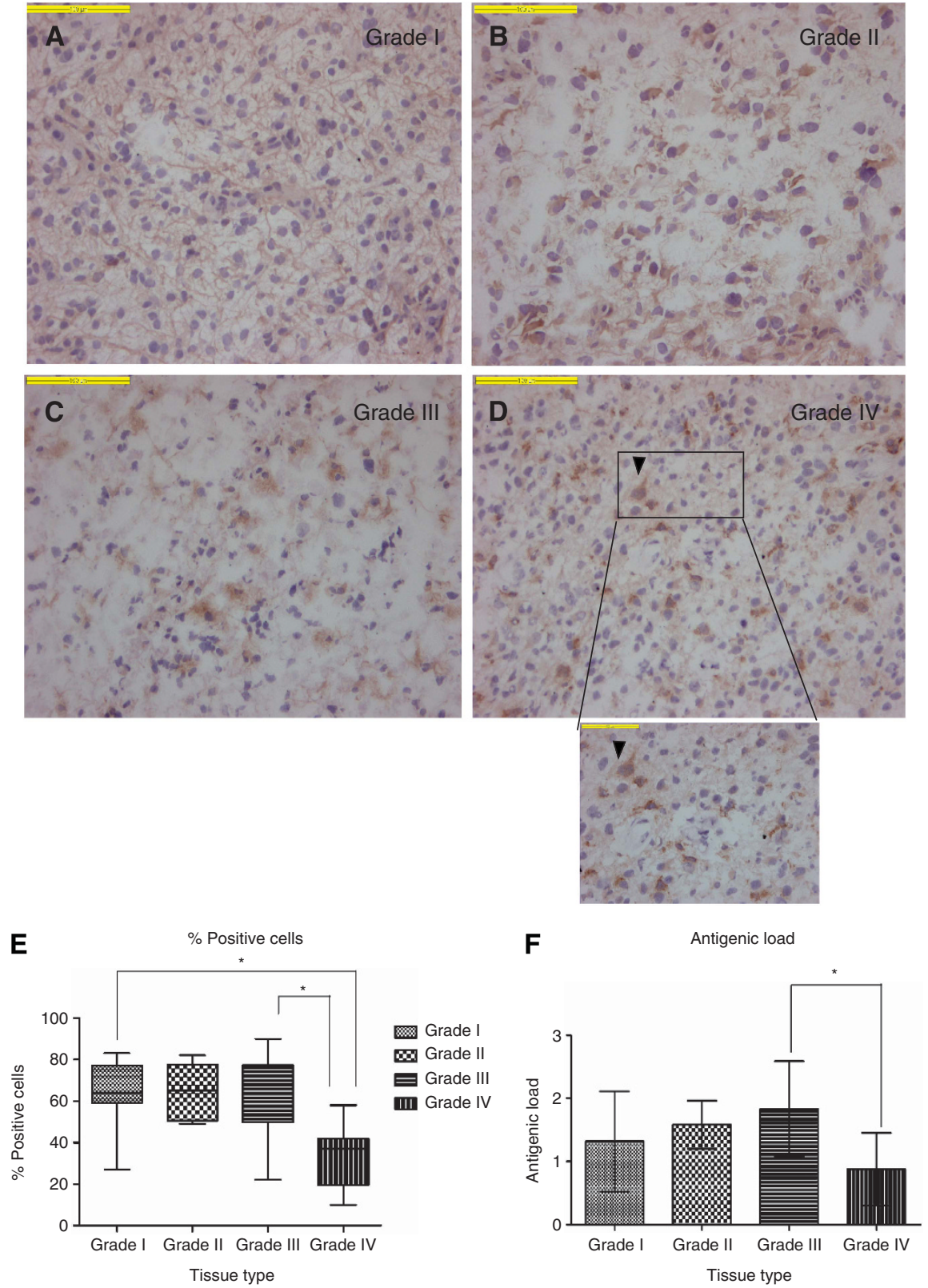

Figure 1. Specimens of human astrocytoma grades I-IV, immunohistochemically stained for FPR1, percentage of positive cells and antigenic load. Micrographs A-D show astrocytic tumours with WHO grades I-IV, respectively. In the low-grade tumours (A and B), diffuse staining can be observed. In the high-grade tumours (C and D), the staining intensity is much less homogeneous. Bars $=100 \mu \mathrm{m}$. (E) Percentages of positive tumour cells in the various grades of astrocytic tumours (dark line in the box refers to the median, whiskers indicate the $95 \% \mathrm{Cl}$ ). The lowest percentage relative to the other three grades is found in glioblastomas $\left({ }^{*} P<0.05\right)$. ( $\left.F\right)$ Antigenic load, the glioblastoma group has the smallest values relative to lower grades $\left({ }^{*} P<0.05\right)$.

U937-FPR cells in a migration and proliferation assays, respectively.

CHIPS inhibited up to $81 \%$ of U937-FPR cells migrating towards fMLF (Figure 4A), whereas chemotaxis towards DMEM/0.5\% BSA was not affected (data not shown). At $10 \mu \mathrm{g} \mathrm{ml}^{-1}$, CHIPS inhibited $83 \%$ of U937-FPR cells migrating towards fMMYALF $\left(10^{-10_{-}}\right.$ $10^{-7} \mathrm{M}$ ) (Figure 4B) and $80 \%$ of U937-FPR cells towards fMLKLIV $\left(10^{-10}-10^{-7} \mathrm{M}\right)$ (Figure $\left.4 \mathrm{C}\right)$. The migration of U87 cells towards fMLF $\left(10^{-7} \mathrm{M}\right)$ was 2.5 -fold higher relative to the control medium (Figure $4 \mathrm{D}$ ). Ten $\mu \mathrm{g} \mathrm{ml}^{-1}$ of CHIPS completely abolished the migration of U87 cells towards $10^{-7} \mathrm{M}$ fMLF (Figure 4D). However, formylated peptides did not induce the proliferation of U87 cells with formylated peptides (data not shown).
CHIPS inhibits phosphorylation of key elements in the downstream pathway of FPR1. The pronounced effects of formylated peptides on calcium mobilisation and cell migration and their inhibition by CHIPS in U87 astrocytoma cells encouraged us to investigate the downstream FPR1 pathway in more detail. Western blot analysis showed that increasing concentrations of fMLF induced the activation of Akt and ERK1/2 in U87 astrocytoma cells (Figure 5A, lanes 2-4). Akt and ERK1/2 phosphorylation were inhibited by CHIPS (Figure 5A, lanes 5-7).

CHIPS inhibits the upregulation of VEGF-A in fMLF-stimulated U87cells. We investigated the ability of U87 cells to excrete VEGF-A upon stimulation with $\mathrm{PMLF}$ and tested whether this 

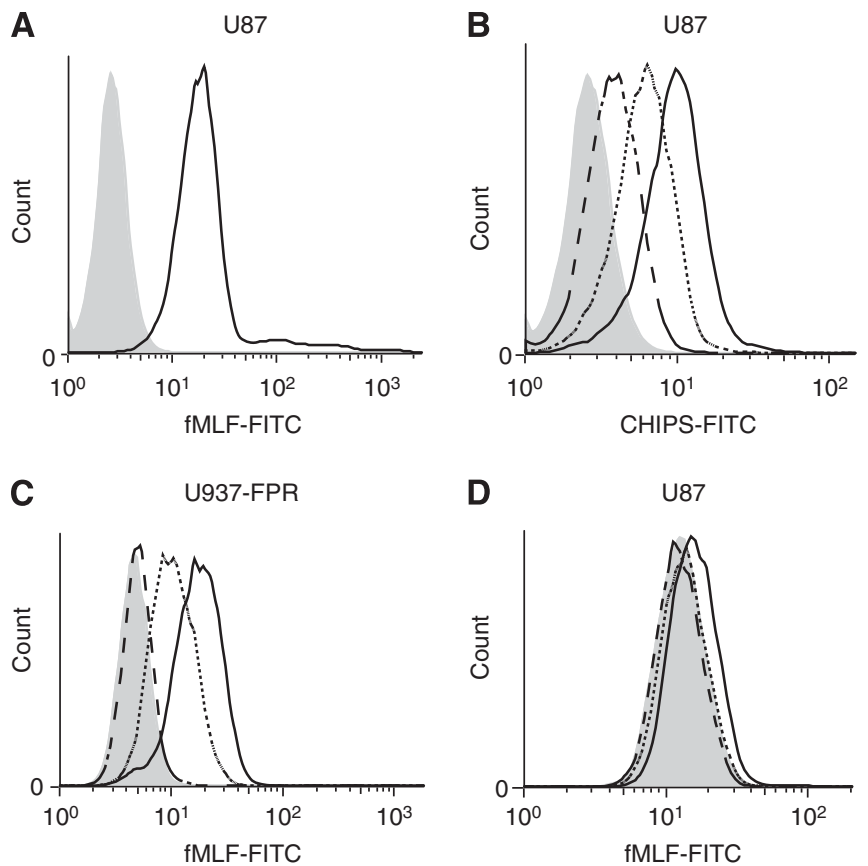

Figure 2. fMLF and CHIPS binding to FPR1 on U87 astrocytoma cell line. (A) Mean fluorescence intensity of FPR1-expressing U87 cells incubated with FMLF-FITC (continuous black line) and control-stained cells (grey histogram). (B) Mean fluorescence intensity of U87 cells incubated with $3 \mu \mathrm{g} \mathrm{ml}^{-1}$ CHIPS-FITC (dashed line), $10 \mu \mathrm{g} \mathrm{ml}^{-1} \mathrm{CHIPS}$ FITC (continuous grey line) and $20 \mu \mathrm{g} \mathrm{ml}^{-1}$ CHIPS-FITC (continuous black line) and baseline staining (grey histogram). (C and D) Blocking of FMLF-FITC binding to U87 and control cells U937-FPR by preincubating cells with CHIPS. Graphs depict mean fluorescence intensity of U87 (C) and U937-FPR (D) cells when preincubated with $1 \mu \mathrm{g} \mathrm{ml}^{-1}$ CHIPS (dotted line) or $100 \mu \mathrm{g} \mathrm{ml}^{-1}$ CHIPS (dashed line).

could be inhibited by CHIPS. The levels of VEGF-A excretion reached by control cells was $5174 \mathrm{pg} \pm 1323$ per $10^{6}$ cells; the average value of control cells was set at $100 \%$. After $24 \mathrm{~h}$ of incubation with fMLF, U87 cells showed on an average, $25 \pm 9 \%$ increased excretion of VEGF-A compared with that of control. In cells preincubated with CHIPS, fMLF could induce only $8 \pm 3 \%$ of VEGF-A excretion $(P<0.04)$ compared with that of control (Figure 5B).

In vivo inhibition of tumour growth by CHIPS. To test the in vivo effects of CHIPS, we subcutaneously implanted U87 cells in NOD-SCID mice. Animals treated with CHIPS showed slight reduction in tumour volume (Figure 6A). CHIPS-treated mice exhibited a $50 \%$ survival of 45 days, whereas untreated animals showed a $50 \%$ survival of 41 days. Median survival of treated animals was higher than untreated animals $(P=0.0019)$ (Figure 6B).

Cleaved caspase-3 expression on animal specimens. We investigated the effects of CHIPS treatment on the induction of apoptosis by analysing the expression of cleaved caspase- 3 in U87 xenografts. Within one and the same tumour, normoxic and hypoxic (Glut1-positive) areas can be distinguished. Between these areas, significant differences were found in the percentages of cleaved caspase- 3 positive cells in treated (normoxic $1.2 \%$ and hypoxic $32.1 \%$ ) and untreated (normoxic $0.6 \%$ and hypoxic $24.5 \%$ ) animals. When comparing normoxic tumour areas between treated and untreated animals, only a small, but significant difference in the percentage of cleaved caspase-3-positive cells was found (treated $0.6 \%$, untreated $1.2 \%(P=0.026)$ ) (Figure 6D). Between the treated and untreated group, no significant differences were observed in the hypoxic area. These results indicate that CHIPS treatment may increase apoptosis induction.

\section{DISCUSSION}

In this study, we investigated FPR1 as an attractive molecular target for the development of novel, urgently needed antiastrocytoma therapeutics. Moreover, we explored the potency of CHIPS in inhibiting FPR1-mediated cellular responses in established astrocytoma cell line U87, a primary patient-tumour derived cell line and in a human xenograft mouse model.

Our immunohistochemical analysis showed high FPR1 expression in all grades of astrocytomas (I-IV). Despite that relevance for FPR1 targeting is likely to be greater in higher grade astrocytomas defined by necrotic areas (Louis et al, 2007), low grade tumours can also acquire angiogenic profiles comparable with glioblastomas (Sie et al, 2010). This indicates that antagonists of FPR1 could be a valuable treatment option for all grades of astrocytoma patients and might be of relevance also for low grade astrocytomas, especially when their location precludes complete resection (Suchorska et al, 2011).

In this study, we found that mitochondrial peptides fMMYALF and AMLKLIV, that are normally released from necrotic cells, exhibit FPR1-mediated calcium flux on FPR1-transfected U937 cell line, but also on U87. This shows for the first time that mitochondrial peptides released by necrotic cells serve as human ligands for FPR1 expressed on human astrocytoma cells. In addition, we demonstrated that the patient-derived astrocytoma grade IV cell line expressed functional FPR1, as we could stimulate calcium mobilisation with fMLF and inhibit this with CHIPS. This confirms the potential clinical relevance of our findings.

Calcium mobilisation initiated by GPCR activation leads to the induction of cell migration. Indeed, in a study of Yao et al (2008b), FPR1 present on CD133 + astrocytoma stem cells could be activated by bacteria or host-derived chemotactic agonists and lead to intracellular calcium mobilisation and tumour cell chemotaxis. Consequently, in our study, CHIPS treatment inhibited the migration on both U87 astrocytoma cells and U937-FPR cells. Taken together, these results indicate that CHIPS is a promising agent to inhibit FPR1 activation on human astrocytoma cells and decrease cell migration, which is an important biological feature of high grade astrocytomas.

The first study investigating the effect of FPR1 inhibition on tumourigenicity of U87 astrocytoma cells was performed in an immunodeficient mouse model. In this model, short interfering RNA (siRNA) against FPR1 markedly reduced the tumourigenic potential of U87 cells (Zhou et al, 2005). This study provided a rationale for targeting the FPR1 in astrocytoma models; however, applying these results in the clinical practice remains challenging due to the problems with delivering siRNA to the tumour cells. In our study, we tested CHIPS, administered intraperitoneally, for its potential to inhibit U87 tumour growth in NOD-SCID mice. Although biological effects in terms of differences in tumour volumes could be observed, these were not significant. Notably, animals in the control group reached the human endpoint earlier compared with treated animals $(P=0.0019)$, proving that CHIPS treatment in vivo prolongs the survival of U87 tumour-bearing mice.

CHIPS is very selective, as it does not affect other chemoattractant receptors present on neutrophils such as the FPR-like 1, complement factor $3 \mathrm{a}$ receptor $(\mathrm{C} 3 \mathrm{aR})$, interleukin (IL) 8 receptor $\alpha$ (IL-8RA) and $-\beta$ (IL-8RB), leukotriene B4 (LTB4) receptors, and the platelet activating factor (PAF) receptor (Postma et al, 2004). Compared with other well known FPR1 inhibitors like cyclosporin 

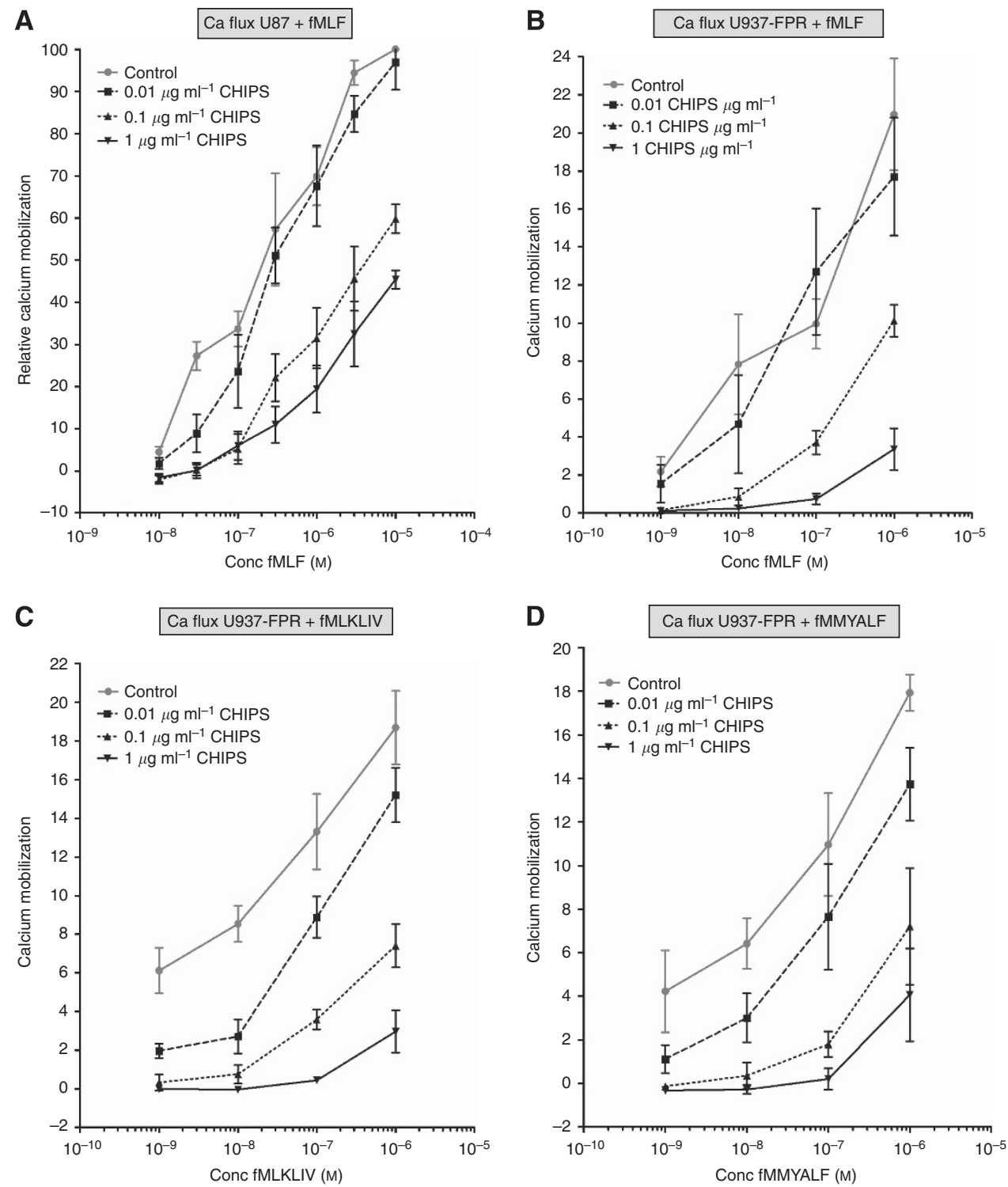

Figure 3. Inhibitory effects of CHIPS on formylated peptides-induced calcium mobilisation in U87 and U937-FPR cell lines. (A) Inhibition of intracellular calcium mobilisation by CHIPS in U87 cells stimulated with fMLF. (B) Inhibition of intracellular calcium mobilisation by CHIPS in U937FPR cells stimulated with fMLF (C) with fMLKLIV and (D) fMMYALF. Data are displayed as percentage of maximal calcium mobilization.

Abbreviation: Conc, concentration.

$\mathrm{H}$ and boc-PLPLP, the affinity and the potency of CHIPS to bind and block FPR1 is 1000-fold higher (Haas et al, 2004a).

The pharmacokinetics and toxicity of CHIPS as an antiinflammatory drug were investigated in a small phase 1 study. In that study, circulating anti-CHIPS antibodies induced toxicities upon intravenous administration of CHIPS due to pre-existing antibodies (Haas et al, unpublished data). As the active site for inhibition of FPR1 is located at the N-terminus and is not dependent on $3 \mathrm{D}$-structural conformations, we recently constructed a 6-amino-acid N-terminal peptide that displays CHIPS activity. Although the peptide is less potent than CHIPS, it was still able to inhibit fMLF-induced neutrophil response (Haas et al, 2004b). Therefore, it represents a good candidate for further development as a clinical FPR1 inhibitor. The development of peptides can easily overcome problems with antigenicity, and peptidomimetics can open the way to enhanced stability in such peptides.

Targeting FPR1 with CHIPS has a relevant clinical rationale due to its interaction with other pathways involved in shaping the aggressive phenotype of glioblastoma. For example, the cross-talk of FPR1 with epidermal growth factor receptor (EGFR) exacerbates the malignant properties of tumour cells like chemotaxis and proliferation. Moreover, knocking down both receptors yielded to superior effects compared with the depletion of either one (Huang et al, 2007). FPR1 activation was also shown to affect VEGF-A and interleukin 8 (IL-8) production by glioblastoma cells after intracranial implantation in nude mice, as tumours formed by cells with silenced FPR1 showed less active angiogenesis, lower expression of both angiogenic factors and reduced tumour volumes (Yao et al, 2008a). Although we could not find any in vitro differences in PMLF-induced U87 cell proliferation as compared with controls, we did find an inhibitory effect of CHIPS on VEGFA excretion in fMLF-induced U87 cell line. This interaction remains particularly interesting in the context of a recent Food and Drug Administration (FDA) approval of bevacizumab (monoclonal antibody against VEGF-A) for the treatment of recurrent grade IV astrocytomas. The approval was based on the results of 

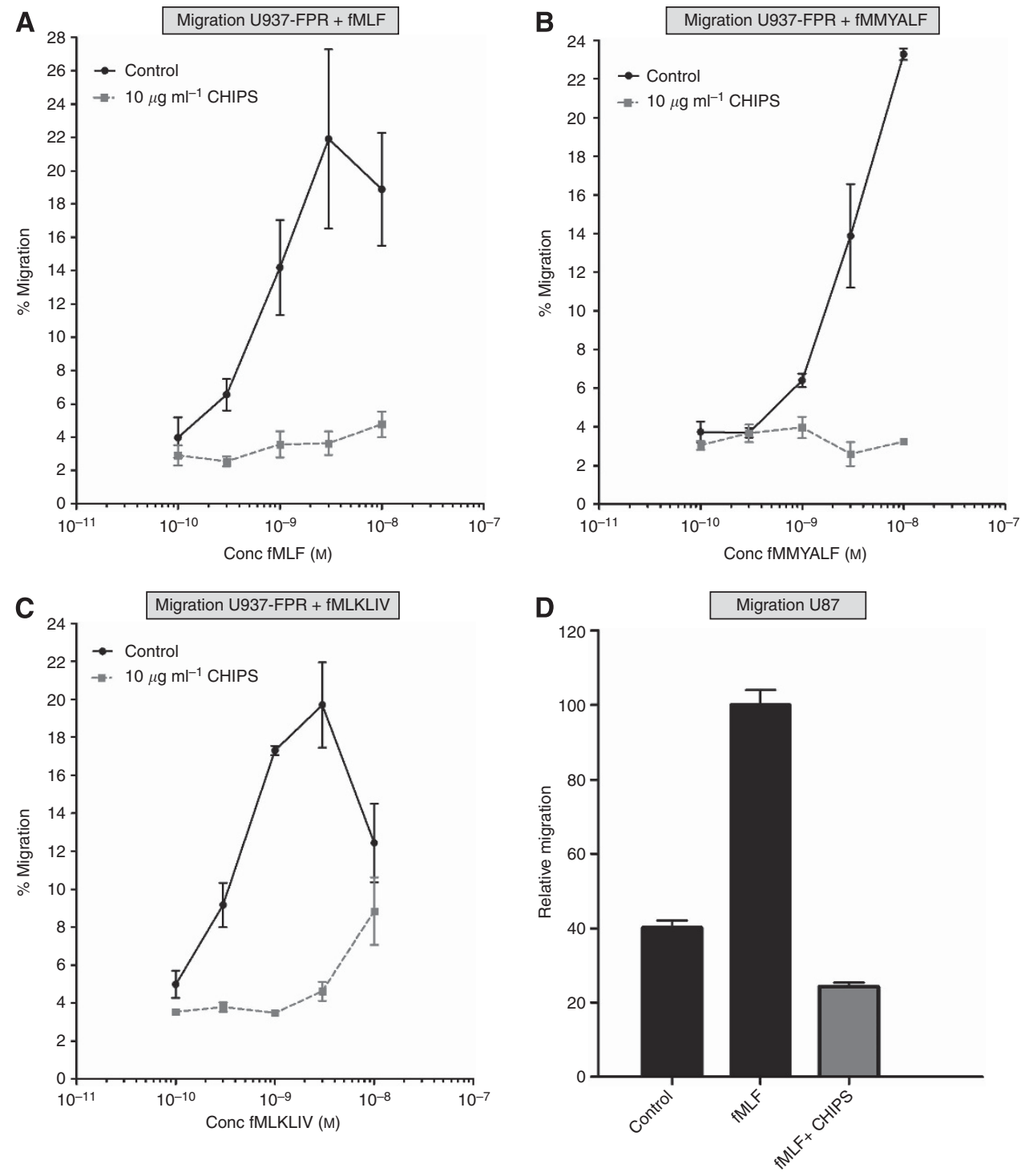

Figure 4. Inhibitory effects of CHIPS on formylated peptides-induced cell migration in U87 and U937-FPR cell lines. (A) The inhibition of cell migration by $10 \mu \mathrm{g} \mathrm{ml}^{-1}$ CHIPS in U937-FPR cells stimulated with $\mathrm{fMLF}\left(10^{-7} \mathrm{M}\right)(\mathrm{B})$ with $\mathrm{PMMYALF}$ and $(\mathrm{C})$ with $\mathrm{fMLKLIV} \mathrm{peptides}\left(10^{-10}-10^{-7} \mathrm{M}\right)$. (D) Inhibition of cell migration by $10 \mu \mathrm{g} \mathrm{ml}^{-1}$ CHIPS in U87 cells stimulated with $10^{-7} \mathrm{M} \mathrm{fMLF}$. Data are presented as percentage of migrated cells as compared with that of the positive control.
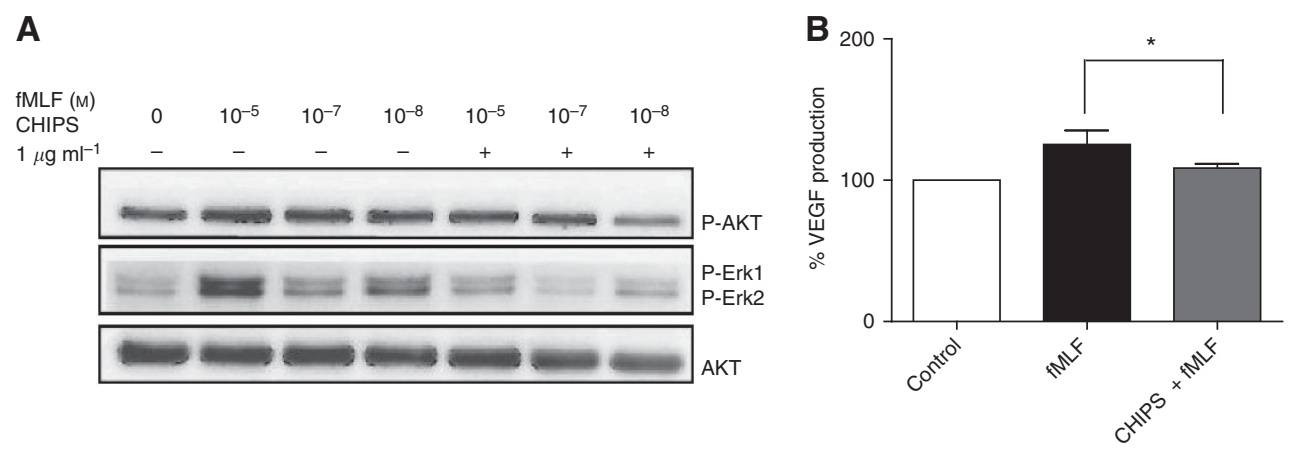

Figure 5. Inhibitory effects of CHIPS on fMLF-induced phosphorylation of Akt, ERK1/2 and total Akt (Western blot) and on VEGF-A excretion (ELISA) in U87 cells (A) Representative immunoblot showing the effects of CHIPS on fMLF-induced phosphorylation of Akt, ERK1/2 and total Akt when incubated with fMLF in U87 cells. (B) Levels of VEGF-A protein in supernatants of U87 cells. Cells were preincubated with or without $10 \mu \mathrm{g} \mathrm{ml}^{-1} \mathrm{CHIPS}$ and treated with $\mathrm{fMLF}\left(10^{-7} \mathrm{M}\right)$ or culture medium (control) for $24 \mathrm{~h}$. Cells preincubated with CHIPS, produced significantly lower VEGF-A excretion upon fMLF treatment compared with fMLF-treated cells without CHIPS preincubation ( $\left.{ }^{\star} P<0.04\right)$. 

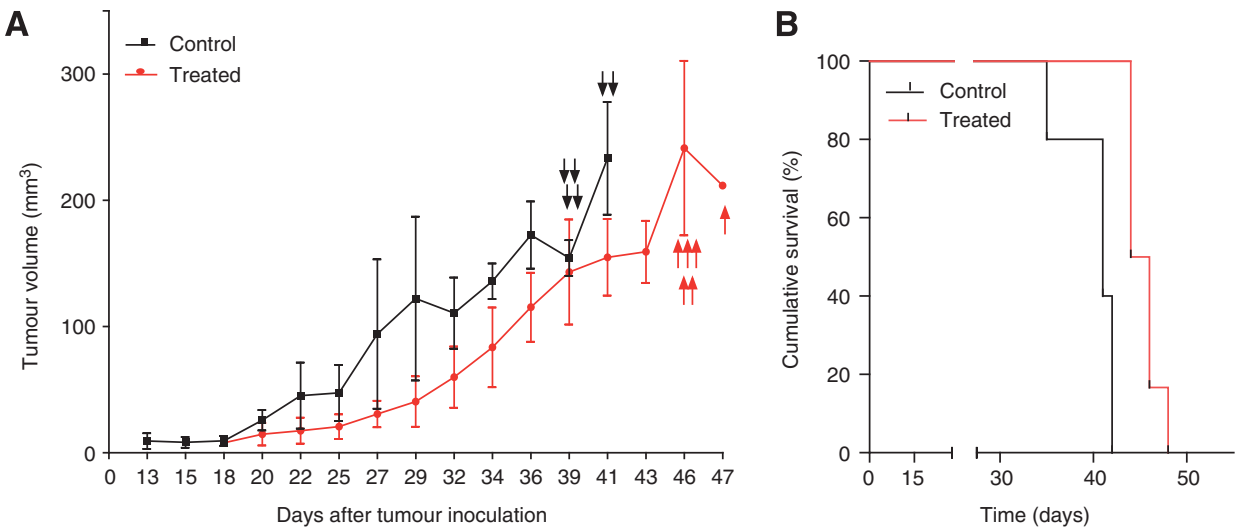

C
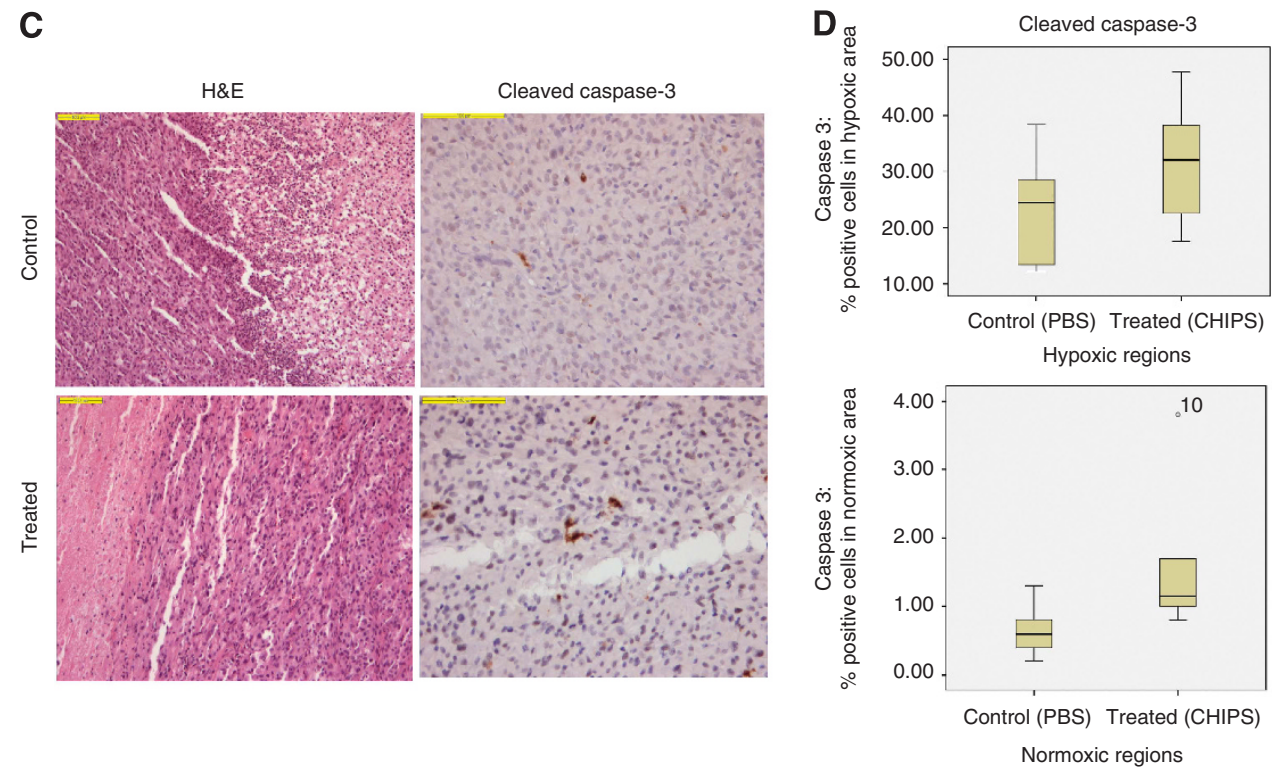

Figure 6. Tumour formation and progression in xenografts. U87 cells $\left(5 \times 10^{6}\right.$ in $100 \mu$ l of PBS) were subcutaneously implanted in the right flank of preirradiated NOD-SCID mice. (A) Tumour growth in U87 subcutaneous xenografts treated with placebo (PBS) or CHIPS was examined. Treated animals received daily injections of $1.2 \mathrm{mg} \mathrm{ml}^{-1}$ CHIPS in $100 \mu \mathrm{l}$ PBS whereas the control animals received daily equal volumes of PBS. Tumour size is expressed as the mean volume in $\mathrm{mm}^{3}$ and the arrows indicate killed animals as they reached the humane endpoint (volume $>2000 \mathrm{~mm}^{3}$ ). (B) Survival of tumour bearing mice. Control animals exhibited significantly lower survival compared with treated animals ( $\left.{ }^{*} P=0.0019\right)$. (C) H\&E and cleaved caspase-3 staining in normoxic regions of control and treated animals. (D) Quantification of cleaved caspase-3 staining in hypoxic and normoxic regions of tumours treated with PBS or CHIPS. Treated animals exhibited slight but significantly higher cleaved caspase- 3 staining in normoxic areas compared with untreated animals $\left({ }^{\star} P=0.026\right)$.

two phase II clinical trials, which showed that bevacizumab reduced tumour size in part of the astrocytoma patients (Vredenburgh et al, 2007; Friedman et al, 2009). At the same time, controversies arose, based on a study in 37 patients suggesting that this antiangiogenic drug may alter the recurrence pattern of malignant gliomas (Norden et al, 2008). Anti-VEGF therapy can facilitate co-option of normal vasculature and tumour invasion, potentially leading to a more aggressive tumour phenotype (Rubenstein et al, 2000; Kunkel et al, 2001; Du et al, 2008). To improve current antiangiogenic therapy in astrocytoma and potentially other tumours, it will be crucial to identify pathways that simultaneously block perivascular invasion as well as angiogenesis.

In summary, mitochondrial peptides present in necrotic tumour material serve as activating ligands for FPR1 on tumour cells. Moreover, CHIPS specifically inhibits cell activation and migration via FPR1 on U87 astrocytoma cells and increases the survival of U87-tumour bearing mice. In addition, FPR1 is present on all grades of human astrocytoma and induces calcium mobilisation in a patient-derived astrocytoma cell line when stimulated with fMLF. We conclude that this receptor constitutes a clinically relevant target. Therefore, further development of CHIPS derivatives might well provide a major contribution to current astrocytoma therapy.

\section{ACKNOWLEDGEMENTS}

This work was supported by the Dutch Cancer Society (grant number RUG 2010-4622) and Vanderes Foundation.

\section{REFERENCES}

de Haas CJ, Veldkamp KE, Peschel A, Weerkamp F, Van Wamel WJ, Heezius EC, Poppelier MJ, Van Kessel KP, van Strijp JA (2004) Chemotaxis inhibitory protein of Staphylococcus aureus, a bacterial antiinflammatory agent. J Exp Med 199: 687-695.

de Vries EG, Meijer C, Timmer-Bosscha H, Berendsen HH, de Leij L, Scheper RJ, Mulder NH (1989) Resistance mechanisms in three human small cell lung cancer cell lines establishedfrom one patient during clinical follow-up. Cancer Res 49: 4175-4178. 
Du R, Lu KV, Petritsch C, Liu P, Ganss R, Passegue E, Song H, Vandenberg S, Johnson RS, Werb Z, Bergers G (2008) HIF1 $\alpha$ induces the recruitment of bone marrow-derived vascular modulatory cells to regulate tumor angiogenesis and invasion. Cancer Cell 13: 206-220.

Durstin M, Gao JL, Tiffany HL, McDermott D, Murphy PM (1994) Differential expression of members of the $\mathrm{N}$-formylpeptide receptor gene cluster in human phagocytes. Biochem Biophys Res Commun 201: 174-179.

Friedman HS, Prados MD, Wen PY, Mikkelsen T, Schiff D, Abrey LE, Yung WK, Paleologos N, Nicholas MK, Jensen R, Vredenburgh J, Huang J, Zheng M, Cloughesy T (2009) Bevacizumab alone and in combination with irinotecan in recurrent glioblastoma. J Clin Oncol 27: 4733-4740.

Haas PJ, de Haas CJ, Kleibeuker W, Poppelier MJ, van Kessel KP, Kruijtzer JA, Liskamp RM, van Strijp JA (2004a) N-terminal residues of the chemotaxis inhibitory protein of Staphylococcus aureus are essential for blocking formylated peptide receptor but not C5a receptor. J Immunol 173: 5704-5711.

Haas PJ, de Haas CJ, Kleibeuker W, Poppelier MJ, van Kessel KP, Kruijtzer JA, Liskamp RM, van Strijp JA (2004b) N-terminal residues of the chemotaxis inhibitory protein of Staphylococcus aureus are essential for blocking formylated peptide receptor but not C5a receptor. J Immunol 173: 5704-5711.

Huang J, Chen K, Chen J, Gong W, Dunlop NM, Howard OM, Gao Y, Bian XW, Wang JM (2010) The G-protein-coupled formylpeptide receptor FPR confers a more invasive phenotype on human glioblastoma cells. $\mathrm{Br} \mathrm{J}$ Cancer 102: 1052-1060.

Huang J, Hu J, Bian X, Chen K, Gong W, Dunlop NM, Howard OM, Wang JM (2007) Transactivation of the epidermal growth factor receptor by formylpeptide receptor exacerbates the malignant behavior of human glioblastoma cells. Cancer Res 67: 5906-5913.

Kuijlen JM, Mooij JJ, Platteel I, Hoving EW, van der Graaf WT, Span MM, Hollema H, den Dunnen WF (2006) TRAIL-receptor expression is an independent prognostic factor for survival in patients with a primary glioblastoma multiforme. J Neurooncol 78: 161-171.

Kunkel P, Ulbricht U, Bohlen P, Brockmann MA, Fillbrandt R, Stavrou D, Westphal M, Lamszus K (2001) Inhibition of glioma angiogenesis and growth in vivo by systemic treatment with a monoclonal antibody against vascular endothelial growth factor receptor-2. Cancer Res $\mathbf{6 1}$ : 6624-6628.

Liao D, Corle C, Seagroves TN, Johnson RS (2007) Hypoxia-inducible factor$1 \alpha$ is a key regulator of metastasis in a transgenic model of cancer initiation and progression. Cancer Res 67: 563-572.

Louis DN, Ohgaki H, Wiestler OD, Cavenee WK, Burger PC, Jouvet A, Scheithauer BW, Kleihues P (2007) The 2007 WHO classification of tumours of the central nervous system. Acta Neuropathol 114: 547.

Macheda ML, Rogers S, Best JD (2005) Molecular and cellular regulation of glucose transporter (GLUT) proteins in cancer. J Cell Physiol 202: 654-662.

Miller CR, Perry A (2007) Glioblastoma. Arch Pathol Lab Med 131: 397-406.

Norden AD, Young GS, Setayesh K, Muzikansky A, Klufas R, Ross GL, Ciampa AS, Ebbeling LG, Levy B, Drappatz J, Kesari S, Wen PY (2008) Bevacizumab for recurrent malignant gliomas: efficacy, toxicity, and patterns of recurrence. Neurology 70: 779-787.
Postma B, Poppelier MJ, van Galen JC, Prossnitz ER, van Strijp JA, de Haas CJ, van Kessel KP (2004) Chemotaxis inhibitory protein of Staphylococcus aureus binds specifically to the C5a and formylated peptide receptor. $J$ Immunol 183: 6569-6578.

Rabiet MJ, Huet E, Boulay F (2005) Human mitochondria-derived $\mathrm{N}$-formylated peptides are novel agonists equally active on FPR and FPRL1, while Listeria monocytogenes-derived peptides preferentially activate FPR. Eur J Immunol 35: 2486-2495.

Rubenstein JL, Kim J, Ozawa T, Zhang M, Westphal M, Deen DF, Shuman MA (2000) Anti-VEGF antibody treatment of glioblastoma prolongs survival but results in increased vascular cooption. Neoplasia 2: 306-314.

Schiffmann E, Corcoran BA, Wahl SM (1975) N-formylmethionyl peptides as chemoattractants for leucocytes. Proc Natl Acad Sci USA 72: 1059-1062.

Sie M, de Bont ES, Scherpen FJ, Hoving EW, den Dunnen WF (2010) Tumour vasculature and angiogenic profile of paediatric pilocytic astrocytoma; is it much different from glioblastoma? Neuropathol Appl Neurobiol 36: 636-647.

Sie M, Wagemakers M, Molema G, Mooij JJ, de Bont ES, den Dunnen WF (2009) The angiopoietin 1/angiopoietin 2 balance as a prognostic marker in primary glioblastoma multiforme. J Neurosurg 110: 147-155.

Suchorska B, Ruge M, Treuer H, Sturm V, Voges J (2011) Stereotactic brachytherapy of low-grade cerebral glioma after tumor resection. Neuro Oncol 13: 1133-1142.

Vivanco I, Sawyers CL (2002) The phosphatidylinositol 3-Kinase AKT pathway in human cancer. Nat Rev Cancer 2: 489-501.

Vredenburgh JJ, Desjardins A, Herndon 2nd JE, Marcello J, Reardon DA, Quinn JA, Rich JN, Sathornsumetee S, Gururangan S, Sampson J, Wagner M, Bailey L, Bigner DD, Friedman AH, Friedman HS (2007) Bevacizumab plus irinotecan in recurrent glioblastoma multiforme. J Clin Oncol 25: 4722-4729.

Wen PY, Kesari S (2008) Malignant gliomas in adults. N Engl J Med 359: 492-507.

Yao XH, Ping YF, Chen JH, Chen DL, Xu CP, Zheng J, Wang JM, Bian XW (2008a) Production of angiogenic factors by human glioblastoma cells following activation of the G-protein coupled formylpeptide receptor FPR. J Neurooncol 86: 47-53.

Yao XH, Ping YF, Chen JH, Xu CP, Chen DL, Zhang R, Wang JM, Bian XW (2008b) Glioblastoma stem cells produce vascular endothelial growth factor by activation of a G-protein coupled formylpeptide receptor FPR. J Pathol 215: 369-376.

Zhou Y, Bian X, Le Y, Gong W, Hu J, Zhang X, Wang L, Iribarren P, Salcedo R, Howard OM, Farrar W, Wang JM (2005) Formylpeptide receptor FPR and the rapid growth of malignant human gliomas. J Natl Cancer Inst 97: $823-835$.

Zlotnik A (2006) Chemokines and cancer. Int J Cancer 119: 2026-2029.

This work is published under the standard license to publish agreement. After 12 months the work will become freely available and the license terms will switch to a Creative Commons AttributionNonCommercial-Share Alike 3.0 Unported License.

Supplementary Information accompanies this paper on British Journal of Cancer website (http://www.nature.com/bjc) 